\title{
THE USE OF INTRAOPERATIVE NEUROPHYSIOLOGICAL MONITORING TECHNIQUES IN SUPRATENTORIAL BRAIN SURGERIES
}

\author{
George H. Ibrahim, Hisham A. Simry, Ahmed M. Darwish, Salah M. Hamada and \\ Sameh M. Hefni.
}

Department of Neurosurgery, Faculty of Medicine, Ain

Shams University.

\section{Corresponding Author:}

George Halim Ibrahim Korkar

Phone No.: (+2 01227021400

E-mail:

georgehalim@med.asu.edu.eg

Received : 20/4/2020

Accepted: $13 / 5 / 2020$

Online ISSN: 2735-3540

\begin{abstract}
:
Background: In patients with lesions located around or within eloquent motor or speech cortex, surgical resection is a challenge; because of high risk of development of postoperative deficits and incomplete resection. intraoperative neurophysiological monitoring (IONM) paves the way to complete resection in these patients along with functional preservation postoperative.
\end{abstract}

Aim of the study: To assess the utility and effectiveness of IONM techniques in resection of lesions located in eloquent motor speech cortex (intrinsic brain tumors and epileptic zones), regarding the extent of resection and functional preservation postoperative.

Patients and Methods: we conducted prospective observational study on 30 patients with different types of lesions located within eloquent motor or speech cortex, by the use of low frequency direct cortical and subcortical stimulation technique,12 patients were operated under general anaesthesia and 18 patients were operated by awake craniotomy technique.All patients were assessed clinically and do MRI brain with contrast and FLAIR study postoperatively, those who developed new onset neurological deficits were assessed at 3 months postoperative.

Results: complete resection was achieved in 17 patients (56.6\%). Immediate postoperative new onset or worsened neurological deficits were encountered in 18 patients (60\%), however 3 months postoperative the deficits were persistent only in 4 patients (13.3\%).

Conclusion: direct cortical stimulation is an easy safe and reliable method for detection of cortical and subcortical functionally eloquent structures during resection of brain tumors and epileptic lesions

Keywords: eloquent cortex, intraoperative neurophysiology, direct cortical stimulation, awake craniotomy.

\section{INTRODUCTION:}

In patients with intra-axial brain tumors and epileptic focuses located around or within eloquent motor or speech cortex, surgical resection is a challenge; because of high risk of development of postoperative neurological deficits along with incomplete resection, so most of these lesions in the past used to be managed conservatively ${ }^{(1)}$.

Functional brain mapping (FBM) is routinely accomplished through the integration of various techniques. Noninvasive means to localize structural pathology center on high-resolution brain magnetic resonance imaging (MRI) along with tractography and 
fMRI, with invasive means either preoperative or intraoperative neurophysiological mapping techniques ${ }^{(2)}$.

Through the last 50 years, the use of intraoperative neurophysiological monitoring techniques became essential for mapping and monitoring of the eloquent cortex. Direct cortical stimulation for Motor mapping can be done under general anesthesia either with low frequency bipolar cortical stimulation or monopolar high frequency cortical stimulation, while direct cortical stimulation for language mapping necessitates operating by awake craniotomy technique $^{(3)}$.

In this study, we studied the efficacy of the direct cortical subcortical stimulation mapping techniques regarding extent of resection and functional preservation in intrinsic brain tumors and epileptic pathologies located with eloquent motor and language areas.

\section{PATIENTS AND METHODS:}

A prospective observational cohort of 30 patients with intrinsic brain tumor or epileptic zones located in eloquent motor or language areas were operated between August 2017 and March 2020.

Patients were included in the study if all preoperative radiological, electrophysiological, functional imaging suggested the presence of lesion within eloquent motor or language areas, and were found to have intact neurological examination or mild deficits, patients excluded from the study were children less than 10 years of age, patients with severe motor weakness or severe dysphasia, informed consent was taken form all patients enrolled in our study.

Perirolandic lesions were operated under general anaesthesia by TIVA protocol, while lesions located in speech areas (inferior frontal, medial frontal, temporal, inferior parietal, insular) were operated under awake conditions with monitored sedation technique with scalp block and local anaethesia along the incision line. In patients with brain tumors, a wide craniotomy flap was done to expose motor and speech centers (positive mapping technique), while in patients operated for epilepsy surgery a small craniotomy flap centered over the lesion was done (negative mapping technique).

\section{a) Motor mapping under general anesthesia for Gliomas and epilepsy surgery:}

Before the patient was draped, subdermal needle electrodes were inserted into 12 to 16 muscles chosen to provide broad coverage of the appropriate body region.

Bipolar low frequency direct cortical stimulation is used, with bipolar probe, distance between tips is $1 \mathrm{~cm}$, to deliver constant current square waves, with pulse width $1 \mathrm{msec}$, frequency $50-60 \mathrm{~Hz}$, multichannel electromyographic recordings were used for recording from the contralateral upper and lower limbs, following the dural opening, the cortical motor region was stimulated and identified using serially increasing currents in increments of $1 \mathrm{~mA}$, starting from $4 \mathrm{~mA}$ for asleep patients (to maximum of $16 \mathrm{~mA}$ ). Then the same current applied at the cortex was used for the subcortical mapping to localize the descending motor pathways.

Subcortical stimulation mapping was started when the resection was carried to the depth on level with the bottom of a sulcus, serial subcortical stimulation was performed with every advancing 2-3 $\mathrm{mm}$ of resection.

Resection started $1 \mathrm{~cm}$ from the positive site or the nearest sulcus to the positive site (which is nearer), resection was carried out with the intent of a maximal resection until positive subcortical mapping sites were 
reached. At this point the resection was stopped.

In cases in which no response was recorded up to $16 \mathrm{ma}$, either in cortical or subcortical mapping, the mapping was considered negative

We didn't use ECoG in motor mapping under general anesthesia

\section{b) Speech and cognition mapping by awake craniotomy for Gliomas:}

Bipolar low frequency direct cortical stimulation is used, with bipolar probe, distance between tips in $1 \mathrm{~cm}$, to deliver constant current square waves, with pulse width $1 \mathrm{msec}$, frequency $50-60 \mathrm{~Hz}$, Stimulation intensity began with $2 \mathrm{~mA}$

increased to a maximum of $6 \mathrm{~mA}$ (with $0.5 \mathrm{~mA}$ increments) until somatosensory or motor or language response is established, stimulation is applied for 3-4 seconds, Stimulation sites were selected randomly, and all sites were stimulated once before any site was stimulated a second time. Subcortical stimulation was done by using the same current threshold that was also applied during tumor resection, in a backand-forth fashion, Resection was carried out with the intent of a maximal resection until positive cortical or subcortical mapping sites were reached. At this point the resection was stopped, we didn't use ECoG during awake craniotomy for Glioma surgery. The choice of intraoperative tasks depends on the site of the lesion.

c) Awake craniotomy for epilepsy surgery:

The same stimulation parameters used as in awake craniotomy for Gliomas, however intraoperative ECoG is used, because all these patients had intractable epilepsy for long period of time, and moreover we did small craniotomies in case of epilepsy surgery, because lesions were small and no disturbance on gross anatomy was found, so we didn't essentially expose the motor strip nor the Broca s area in cases in which they were not involved. No subcortical white matter stimulation is used because the pathologies were confined to the grey matter

Operative data were collected regarding: Positive or Negative subcortical mapping, maximum intensity used in every case, intraoperative seizures, time spent during mapping process, aoperative vascular injury or injuries to important structures

Postoperatively Functional assessment was done by Assessment of postoperative motor power, speech (fluency and comprehension), and comparing the data with the preoperative assessment data, patients who developed new deficits or worsened deficits postoperative, were followed up for 3 months.

In case of epilepsy surgery, assessment of postoperative seizure control by Engel $\mathrm{s}$ classification,immediately postoperative and after 3 months

Extent of resection was assessment by MRI brain with contrast in all cases within 48 hours after surgery. In cases of diffuse non enhancing gliomas, FLAIR and T2 sequence is used to asses the extent of resection and in cases of high grade tumors, Gadolinium enhanced MRI sequence is used to assess the extent of resection.

\section{RESULTS:}

This study includes 30 patients, 17 males and 13 females, 18 patients $(60 \%)$ were operated by awake craniotomy protocol (15 patients with brain tumors, and 3 patients with non gliomatous epileptic lesions), and 12 patients $(40 \%)$ were operated under general anesthesia (11 patients with brain tumors and 1 patient with FCD). Table 1 and 2 demonstrate patients and lesions characters, table 3 demonstrate intraoperative data .

Immediate postoperative new onset or worsened neurological deficits were 
encountered in our series in 18 patients $(60 \%)$. In patients operated under awake conditions, postoperative new onset or worsened neurological deficit were reported in 10 patients $(55 \%)$, in the form of mild expressive dysphasia (2), severe expressive dysphasia (1), mild mixed dysphasia (3), severe mixed dysphasia (1), contralateral hemiparesis grade 3 (2), and severe contralateral hemiparesis (grade 2) and dysarthria (1). In patients operated under general anesthesia, postoperative deficits were encountered in 8 patients $(66 \%)$, in the form of contralateral weakness grade 4 (2 patients), grade 3 (3 patients), grade 2 along with dysarthria in (3 patients).

In the follow up visit at 3 months postoperative, New onset or worsened neurological deficits were persistent only in 4 patients $(13.3 \%), 3$ of which were mild in the from of mild expressive dysphasia and mild contralateral weakness grade $4 / 5$, and 1 patient was severe in the form of contralateral hemiparesis grade $3 / 5$ and dysarthria.

Among the 10 patients who had preoperative neurological deficits, preoperative neurological deficits improved to become normal by examination in 4 patients $(40 \%)$ and remain stationary in 6 patients $(60 \%)$.

In patients with Non Gliomatous Epielptic zones, seizures control outcome was assessed using Engels classification, all patients were seizures free immediate postoperative. 3 months postoperative 3 patients $(75 \%)$ were Engel class 1a and 1 patient $(25 \%)$ were Engel class $2 \mathrm{a}$.

Complete resection was achieved in 17 patients $(56.6 \%)$ in which no residual signal was detected in flair or contrast images on MRI brain, subtotal resection (less than 10 cc residual) was achieved in 5 patients $(16.6 \%)$, and partial resection (more than 10 cc residual) was done in 8 patients $(26.6 \%)$. Table 4 summarizes the postoperative data
Using the Chi square, we studied the relations and statistical significance between the study variables (anesthesia protocol, positive or negative subcortical mapping, different types of pathologies) and the study outcomes (extent of resection, and functional outcome), and the relation is considered statistically significant if $\mathrm{P}$ value is less than 0.05 .

Complete resection was achieved radiologically in $66.6 \%$ of patients with positive subcortical mapping versus $35.7 \%$ of patients with negative subcortical mapping $(\boldsymbol{P}=\mathbf{0 . 1 1})$. Complete resection was achieved in $50 \%$ of patients operated under awake conditions versus $66.6 \%$ of patients operated under general anesthesia $(P=0.3)$. In Patients with Grade 4 lesions (GBM and metastasis) complete resection was achieved in $41.6 \%$ patients versus $42.8 \%$ of those with diffuse gliomas (grade 2 and 3 ) and $100 \%$ of those with non gliomatous epileptic lesions $(\boldsymbol{P}=\mathbf{0 . 0 9})$.

Early new onset or worsened postoperative neurological deficits were encountered $83 \%$ of patients with positive subcortical mapping versus $42.8 \%$ of those with negative subcortical mapping $(\mathbf{P}=$ 0.03), new onset deficits occurred $55 \%$ of those operated under awake conditions versus $66 \%$ of those operated under general anesthesia $(\boldsymbol{P}=\mathbf{0 . 5})$, and in $58.3 \%$ of the patients with GBM and metastasis versus $64.2 \%$ of those with diffuse gliomas and $50 \%$ of those with epileptic lesions $(\boldsymbol{P}=$ 0.8)

Late neurological deficits persistent after three months, were encountered in $25 \%$ of patients with positive subcortical mapping versus $7 \%$ of those with negative subcortical mapping, however this difference is not statistically significant $(\boldsymbol{P}=\mathbf{0 . 2})$ and were encountered in $11 \%$ of the patients operated under awake conditions versus $16 \%$ in general anesthesia group $(\boldsymbol{P}=\mathbf{0 . 6})$, and in $25 \%$ of the patients with GBM and 
metastasis versus $7 \%$ of those with diffuse gliomas versus 0 in epileptic lesions $(\boldsymbol{P}=$ 0.2). Table 5 summarizes the relation between (subcortical mapping findings, anesthesia protocol, and type of lesion) and (the extent of resection, early and late neurological

deficits.

Table 1: patients data and demographics

\begin{tabular}{|c|c|}
\hline \multicolumn{2}{|c|}{ Table 1: Patient demographics } \\
\hline - $\quad$ Entire cohort & 30 patients \\
\hline - $\quad$ Age & Mean 37 years $(16-72)$ \\
\hline \multicolumn{2}{|l|}{ - $\operatorname{Sex}$} \\
\hline Male & $17(56.6 \%)$ \\
\hline Female & $13(43.33 \%)$ \\
\hline \multicolumn{2}{|l|}{ - $\quad$ Handedness } \\
\hline Right handed & $28(93.3 \%)$ \\
\hline Left handed & $2(6.6 \%)$ \\
\hline \multicolumn{2}{|c|}{ - Clinical presentation } \\
\hline Seizures & $24(80 \%)$ \\
\hline Motor affection & $7(23.3 \%)$ \\
\hline Mild mixed dysphasia & $2(6.6 \%)$ \\
\hline $\begin{array}{ll}\begin{array}{l}\text { Mild } \\
\text { dysphaisa }\end{array} & \text { expressive } \\
\end{array}$ & $1(3.3 \%)$ \\
\hline $\begin{array}{l}\text { Increased intracranial } \\
\text { tension }\end{array}$ & $2(6.6 \%)$ \\
\hline Headache & $5(16.6 \%)$ \\
\hline
\end{tabular}

Table 2: lesion characters

\begin{tabular}{|c|c|}
\hline \multicolumn{2}{|c|}{ Table 2: Lesions characteristics } \\
\hline GBM & $8(26.6 \%)$ \\
\hline Metastsis & $4(13.3 \%)$ \\
\hline $\begin{array}{l}\text { Gliomas Grade } 3 \\
\text { - Anaplastic oligoastrocytoma } \\
\text { - Anaplastic } \\
\\
\text { oligodendroglioma }\end{array}$ & $\begin{array}{l}6(20 \%) \\
5(16.6 \%) \\
1(3.3 \%)\end{array}$ \\
\hline $\begin{array}{c}\text { Glioma Grade } 2 \\
\text { - } \quad \text { astrocytomas } \\
\text { - } \quad \text { oligodendroglioma } \\
\end{array}$ & $\begin{array}{l}8(26.6 \%) \\
4(13.3 \%) \\
4(13.3 \%)\end{array}$ \\
\hline $\begin{aligned} & \text { Epileptic lesions } \\
& \text { - } \text { Focal cortical dysplasia } \\
& \text { - } \text { DNET } \\
& \text { - } \text { Non lesional epilepsy }\end{aligned}$ & $\begin{array}{l}4(13.3 \%) \\
2(6.6 \%) \\
1(3.3 \%) \\
1(3.3 \%)\end{array}$ \\
\hline \multicolumn{2}{|l|}{ Radiological findings } \\
\hline - Site & \\
\hline Left & $23(76.7 \%)$ \\
\hline Right & $7(23.3 \%)$ \\
\hline Frontal & $7(23.3 \%)$ \\
\hline Temporal & $5(16.6 \%)$ \\
\hline Insular & $6(20 \%)$ \\
\hline Perirolandic postcentral & $6(20 \%)$ \\
\hline Perirolandic precentral & $6(20 \%)$ \\
\hline$\bullet \quad$ Size & \\
\hline $\begin{array}{l}\text { Volume } \\
\text { - } \text { GBM and metastasis } \\
\text { Diffuse gliomas (Grade } 2 \\
\text { and 3) }\end{array}$ & $\begin{array}{l}31.6 \quad \mathrm{~cm} 3 \quad(10.5- \\
122)\end{array}$ \\
\hline Maximum diameter & $4.5 \mathrm{~cm}(2.6-7.5)$ \\
\hline
\end{tabular}


George H. Ibrahim, et al.,

Table 3: intraoperative data

\begin{tabular}{|c|c|}
\hline \multicolumn{2}{|c|}{ Table 3: Intraoperative data } \\
\hline • $\quad$ Anaesthesia & \\
\hline $\begin{array}{l}\text { Awake craniotomy } \\
\text { - } \quad \text { Brain tumor } \\
\text { - } \quad \text { Non tumoral Epileptic pathologies }\end{array}$ & $\begin{array}{l}18(60 \%) \\
15(83.3 \%) \\
3(16.7 \%)\end{array}$ \\
\hline $\begin{array}{l}\text { General anesthesia (TIVA) } \\
\text { - } \text { Brain tumors } \\
\text { - } \quad \text { Non tumoral epileptic pathologies }\end{array}$ & $\begin{array}{l}12(40 \%) \\
11(91.6 \%) \\
1(8.4 \%) \\
\end{array}$ \\
\hline \multicolumn{2}{|l|}{ - IONM current intensity } \\
\hline Awake craniotomy & $2.6 \mathrm{ma}(1.5-6)$ \\
\hline General anesthesia & $9 \mathrm{ma}(6-12)$ \\
\hline \multicolumn{2}{|l|}{ - IONM results } \\
\hline $\begin{array}{l}\text { Awake craniotomy } \\
\text { - } \quad \text { Positive cortical mapping } \\
\text { - } \quad \text { Positive subcortical mapping } \\
\end{array}$ & $\begin{array}{l}18(100 \%) \\
8(53.3 \%)\end{array}$ \\
\hline $\begin{array}{l}\text { General anesthesia } \\
\text { - } \quad \text { Positive cortical mapping } \\
\text { - } \quad \text { Positive subcortical mapping }\end{array}$ & $\begin{array}{l}12(100 \%) \\
6(54.4 \%)\end{array}$ \\
\hline \multicolumn{2}{|c|}{ - Duration from dural opening to end of resection } \\
\hline Awake craniotomy & 110 mins $(70-140)$ \\
\hline General anesthesia & 70 mins $(40-100)$ \\
\hline \multicolumn{2}{|l|}{ • Intraoperative seizures } \\
\hline Awake craniotomy & $2(11 \%)$ \\
\hline General anesthesia & $2(16.6 \%)$ \\
\hline Total & $4(13.3 \%)$ \\
\hline \multicolumn{2}{|l|}{ Intraoperative complications } \\
\hline Vascular injury & $1(3.3 \%)$ \\
\hline
\end{tabular}

Table 4: postoperative data

\begin{tabular}{|l|l|}
\hline \multicolumn{3}{|c|}{ Table 4: Postoperative data } \\
\hline Immediate Postoperative New onset neurological deficit \\
\hline Awake craniotomy & $10(55 \%)$ \\
\hline General anesthesia & $8(66 \%)$ \\
\hline Total & $18(60 \%)$ \\
\hline Assessment of postoperative new onset deficits at 3 months postoperative \\
\hline Awake craniotomy & $2(11 \%)$ \\
\hline General anesthesia & $2(16.6 \%)$ \\
\hline Total & $4(13.3 \%)$ \\
\hline $\begin{array}{l}\text { Assessment of Preoperative neurological deficits (10 patients) at } 3 \text { months } \\
\text { postoperative }\end{array}$ \\
\hline Improved to Normal & $4(40 \%)$ \\
\hline Remain stationary & $6(60 \%)$ \\
\hline Extent of resection & $17(56.6 \%)$ \\
\hline Total resection & $5(16.6 \%)$ \\
\hline Subtotal resection & $8(26.6 \%)$ \\
\hline Partial resection & \\
\hline
\end{tabular}


Table 5: the effect of subcortical mapping, anesthesia type, lesion type on the functional and oncological outcome

\begin{tabular}{|c|c|c|c|}
\hline & $\begin{array}{c}100 \% \text { resection } \\
(17 \text { of } 30)\end{array}$ & $\begin{array}{c}\text { Early neurological } \\
\text { deficits } \\
18\end{array}$ & $\begin{array}{c}\text { Late neurological } \\
\text { deficits } \\
4\end{array}$ \\
\hline $\begin{array}{c}\text { Positive subcortical } \\
(12)\end{array}$ & 8 & 10 & 3 \\
$(66.6 \%)$ & $83 \%$ & $25 \%$ \\
\hline Negative subcortical & 5 & 6 & 1 \\
$(14)$ & $(35.7 \%)$ & $42.8 \%$ & 0.2 \\
\hline P value & 0.09 & 0.03 & 2 \\
\hline Awake anesthesia & 9 & 10 & $11 \%$ \\
18 & $50 \%$ & $55 \%$ & 2 \\
\hline General anesthesia & 8 & 8 & 0.6 \\
\hline 12 & $66.6 \%$ & $66 \%$ & 3 \\
\hline P value & 0.3 & 0.5 & $25 \%$ \\
\hline High grade & 5 & 7 & 1 \\
12 & $41.6 \%$ & $58.3 \%$ & $7 \%$ \\
\hline Diffuse low grade & 6 & 9 & 0 \\
\hline 14 & $42.8 \%$ & $64.2 \%$ & 0.2 \\
\hline Epileptic lesions & 4 & 2 & $50 \%$ \\
\hline 4 & $100 \%$ & 0.8 & \\
\hline P value & 0.09 & & \\
\hline
\end{tabular}

\section{DISCUSSION:}

Our study shows that the use of an intraoperative neurophysiological monitoring technniques in supratentorial brain surgeries, either tumor surgery or epilepsy surgery, can certainly constitute an addedvalue for some complex lesions located on eloquent cortex, as it allows some, otherwise non - or barely - operable, patients to become eligible for excision, with minimal risks of functional complications, and good chances total and subtotal excision.

Negative and positive mapping are two different strategies adopted by different surgeons for cortical mapping. Positive mapping implies the application of large craniotomy to expose the motor strip and ventral premotor cortex, even if they are not near to the lesion. Negative mapping implies applying a small craniotomy tailored according to the tumor size, and not essentially expose the motor strip and ventral premotor cortex.

In our study, we adopted the technique of positive mapping in brain tumor surgery, and the negative mapping technique in resective epilepsy surgery.

Duffau et al., adopted the strategy of positive mapping, and demonstrated that the positive mapping allows the surgeon to know the least possible current intensity needed to elicit a motor response and thus decrease the incidence of intraoperative seziures and hence, surgery can be done without the need for ECoG, which makes the procedure more complex. Moreover it is worth noting that such a negative mapping can be dangerous, particularly in non-expert hands, because it can be due to false negative for methodological reason and doesn't gurantee the absence of eloquent sites. Moreover, a positive mapping may also lead to optimize the EOR, since the 
resection can be pursued until eloquent areas are encountered - i.e., enabling to tend towards complete or even "supracomplete" resection when the glioma involves non-eloquent regions ${ }^{(4)}$.

Berger et al. adopted the technique of negative mapping, which implies smaller craniotomy size which protects the brain surface from scarring which could be harmful in the next surgeries, moreover the small size of craniotomy and hence less time consuming and less complications related to craniotomy, this technique implies the usage of ECoG, to be able to increase the current to the maximum instensity to make sure that this area is negative. To our knowledge there is no studies in the literature comparing both techniques. ${ }^{(5)}$

Our results showed that immediate postoperative new onset or worsened neurological deficits occurred in $60 \%$ of the patients, with no significant difference between those operated under awake craniotomy or under general anesthesia (55\% in awake craniotomy patients and 66\% in general anesthesia patients), and hence with no difference between lesions located in language areas and those located in motor areas, we reported also no significant difference in the immediate functional outcome between grade 4 lesions $(58.3 \%)$, diffuse gliomas grade 2 and $3(64.2 \%)$ and non gliomatous epileptic lesions (50\%), however, we found that immediate postoperative new deficits were more associated with positive subcortical mapping $(66.5 \%)$ versus $(35.7 \%)$ in negative subcortical mapping $(\mathrm{P}=0.03)$.

Persistent postoperative deficits after 3 months were encountered in only $13.3 \%$, they were more associated with positive subcortical mapping and grade 4 lesions (however all these associations were not statistically significant) all deficits were mild in the form of mild contralateral weakness grade 4 and mild dysphasia except one patient with contralateral weakness grade 3

Berger et al. in his study of 702 patients with peri-rolandic gliomas who underwent cortical subcortical stimulation mapping revealed new onset or worsened neurological deficits in $30 \%$ of the patients, with persistent neurological deficits after 3 months in only $7.0 \%$ of the entire cohort. Patients with positive subcortical mapping were more likely to develop a new or worsened motor deficit postoperatively ( $45 \%$ vs $19 \%$, respectively, $p<0.001$ ). This difference existed in immediate as well as persistent neurological deficits ${ }^{(6)}$.

In a systematic review about the use of intraoperative stimulation in glioma surgery published in 2012, revealed that Early severe deficits occurred more frequently in patients after resections with stimulation mapping $(36.0 \%)$ than without stimulation mapping (11.3\%), Importantly, event rates subsided to substantially fewer late severe deficits with stimulation mapping (3.4\%) than without stimulation mapping $(8.3 \%)$. This indicates that reversible temporary loss of function of critical brain structures is more frequent with stimulation mapping, but irreversible neurologic damage is more effectively avoided, in comparison with resective surgery, without stimulation mapping ${ }^{(7)}$.

Duffau et al 2005 compared two cohorts of patients with DLGG who underwent surgical resection in the same department in two consecutive periods: in the first period, surgery was performed in 100 patients without mapping; in the second period, resection was achieved in 122 patients with intraoperative cortical stimulation mapping. The percentage of permanent deficit in the first group was $17 \%$ versus $6.5 \%$ in the second ${ }^{(4)}$.

Total resection is defined as the complete removal of the enhanced part of the tumor on T1 gadolinium weighted MRI 
for grade 4 enhancing gliomas and as the complete removal of the FLAIR-weighted MRI abnormalities for diffuse non enhancing grade 2 and 3 gliomas - knowing that a post-operative residual tumoral volume under $10 \pm 5 \mathrm{cc}$ is defined as a subtotal resection ${ }^{(8)}$.

Our study showed that complete resection was achieved in $56.6 \%$ of the patients, subtotal resection in $16.6 \%$ and partial resection in $26.6 \%$, greater extent of resection was more associated with positive subcortical mapping than negative subcortical mapping, no significant difference in EOR was found between those operated under general anesthesia or awake craniotomy, and hence no difference between perirolandic lesions and lesions located in language areas. All non gliomatous epileptic zones showed 100\% resection, while no difference regarding extent of resection between other types of lesions either high or low grade lesions.

Duffau et al in comparing two subgroups of DLGG patients who had surgery in the same department during two consecutive periods, without and then with mapping, $37 \%$ of resections were subtotal and $6 \%$ total in the (no mapping) subgroup versus $50.8 \%$ and $25.4 \%$, respectively, in the second subgroup of patients operated on with cortical subcortical mapping $(\mathrm{P}<$ $0.001)^{(4)}$.

Bello et al. in his series comparing patients with DLGG operated before and after introduction of cortical subcortical mapping techniques revealed the percentage of total and subtotal resections increased from $11 \%$, in the period in which no mapping was available to $69.8 \%$ in the era in which brain mapping techniques were applied. ${ }^{(9)}$

In the same way, in a meta-analysis by Berger et al. on the impact of intraoperative mapping on glioma surgery outcome, based upon over 8000 cases, resections of both high grade gliomas and DLGGs using cortical and subcortical stimulation mapping revealed the percentages of radiologically confirmed gross total resections were $75 \%$ with cortical stimulation mapping versus $58 \%$ without cortical stimulation mapping ${ }^{(7)}$.

Our results showed that the incidence of intraoperative seizures was $13.3 \%$ in the whole cohort, in those operated by awake craniotomy it was $11 \%$, and it was $16.6 \%$ in those under general anesthesia, all seizures were very mild and brief, and aborted only with ice cold ringer, with no neurological sequelae.

In order to decrease the amplitude of current intensity as much as possible and obtain a reliable stimulation mapping during awake craniotomy, we systematically exposed the primary motor cortex and ventral premotor cortex, whatever the location of the lesion. Thanks to a wider bone flap, we reached $100 \%$ of positive mapping with low intensity, by eliciting motor arrest, dysarthria, then afterwards we kept the same intensity for the remainder of the cortical (using further tasks) and subcortical mapping. In general anesthesia cases, the use of TIVA protocol, along with bi-spectral index to keep light sedation during the stimulation, and the use of contralateral multichannel EMG, allowed us to elicit motor response with the lowest possible current intensity.

Duffau et al. in his prospective series of 374 with gliomas operated by awake craniotomy, $80 \%$ presented with seizures, among which $20 \%$ were intractable, the rate of intraoperative seizures was $3.5 \%$, and all seizures were mild and aborted with ice cold ringer lactate, and his results showed that intraoperative seizures were not related to preoperative seizure control or duration, Duffau et al in his series didn't use ECoG, and he applied the same techniques adopted in our series and mentioned above ${ }^{(10)}$. 
Berger et al in his cohort of 840 patients, operated by the use of IONM along with ECOG, reported Stimulation induced seizures occurred in $3 \%$ of patients regardless of the stimulation current and were rapidly terminated with ice-cold Ringer's lactate solution except in 2 patients $^{(5)}$.

Nossek et al. reported that early detection of epileptic activity using ECoG was not associated with the reduction of the incidence of IOS. In fact, it was even associated with an increased risk of IOS (16.5\% with ECoG vs $8.5 \%$ without ECoG). This could be caused by more important stimulation increment when using $\mathrm{ECoG}^{(11)}$.

\section{Conclusion:}

The intraoperative direct electrical bipolar stimulation for cortical and subcortical mapping and monitoring, either under awake condition or under general anesthesia is an easy, reliable, and a safe technique for the detection of both cortical and subcortical functionally eloquent structures during the resection of brain tumors or epileptic zones, and is feasible to be performed routinely during resections in eloquent areas with an extremely low complications and failure rates the corticosubcortical mapping enables us to achieve greater extent of resection of lesions located in eloquent brain areas (previously managed conservatively). This technique is associated increased immediate postoperative new onset neurological deficit, however, decreesed incidence of permanent neurological deficits.

\section{Disclosure:}

The authors confirm that this paper has not been published in its current form or substantialy similar form elsewhere including on a website and also it has not been accepted for publication elsewhere.

The authors disclose no conflict of interests.

\section{REFERENCES:}

1. Lacroix M, Abi-Said D, Fourney DR, Gokaslan ZL, Shi W, DeMonte F, et al. A multivariate analysis of 416 patients with glioblastoma multiforme: Prognosis, extent of resection, and survival. Journal of Neurosurgery. 2001;95(2):190-8

2. Castellano A, Cirillo S, Bello L, Riva M, Falini A. Functional MRI for Surgery of Gliomas. Vol. 19, Current Treatment Options in Neurology. Current Science Inc.; 2017

3. Ottenhausen M, Krieg SM, Meyer B, Ringel F. Functional preoperative and intraoperative mapping and monitoring: Increasing safety and efficacy in glioma surgery. Neurosurgical Focus. 2015;38(1).

4. Duffau H. Intraoperative corticosubcortical stimulations in surgery of lowgrade gliomas [Internet]. Vol. 5, Expert Review of Neurotherapeutics. 2005 [cited 2020 Mar 23]. p. 473-85.

5. Hervey-Jumper SL, Li J, Lau D, Molinaro AM, Perry DW, Meng L, et al. Awake craniotomy to maximize glioma resection: methods and technical nuances over a 27year period. Journal of neurosurgery [Internet]. 2015 Aug 1 [cited 2020 Mar 22]; 123(2):325-39.

6. Han SJ, Morshed RA, Troncon I, Jordan KM, Henry RG, Hervey-Jumper SL, et al. Subcortical stimulation mapping of descending motor pathways for perirolandic gliomas: assessment of morbidity and functional outcome in 702 cases. Journal of neurosuery 2018 Aug 17 [cited 2020 Mar 22]; 131(1):201-8.

7. De Witt Hamer PC, Gil-Robles Grupo Hospitalario Quirón S. Impact of Intraoperative Stimulation Brain Mapping on Glioma Surgery Outcome: A MetaAnalysis Diffuse low-grade gliomas View project ELGGN Imaging View project. Article in Journal of Clinical Oncology [Internet]. 2012 Jul 10 [cited 2020 Mar 22]; 30(20):2559-65. 
8. De Witt Hamer PC, Robles SG, Zwinderman AH, Duffau H, Berger MS. Impact of intraoperative stimulation brain mapping on glioma surgery outcome: A meta-analysis [Internet]. Vol. 30, Journal of Clinical Oncology. 2012 [cited 2020 Mar 23]. p. 2559-65.

9. Bello L, Fava E, Casaceli G, Bertani G, Carrabba G, Papagno C, et al. Intraoperative Mapping for Tumor Resection. Vol. 19, Neuroimaging Clinics of North America. 2009. p. 597-614.
10. Duffau H. A new concept of diffuse (lowgrade) glioma surgery. Vol. 38, Advances and technical standards in neurosurgery. 2012. p. 3-27

11. Nossek E, Matot I, Shahar T, Barzilai O, Rapoport Y, Gonen T, et al. Intraoperative seizures during awake craniotomy: Incidence and consequences: Analysis of 477 patients. Neurosurgery. 2013 Jul;73(1):135-40. 


\section{استخدام تقتيات رصد الفسيولوجيا العصبيه في جراحات المخ فوق الخيمه المخيه}

جورج حليم ابراهيم, هشام عبد السلام سمري, أحمد درويش محمود, صلاح مصطفي حماده, سامح محمد حفني قسم جر احة المخ و الأعصاب, كلية الطب, جامعه عين شمس

الهـف و المقدمه:يمثل استئصسال اورام المخ و البؤر الصر عيه الي تقع بمر اكز المخ المسئوله عن الكلام و الحركه

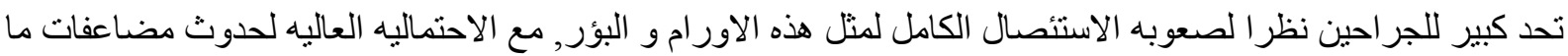

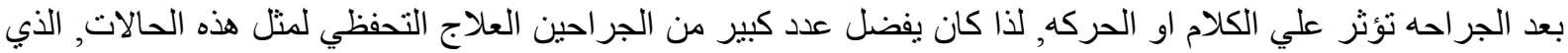

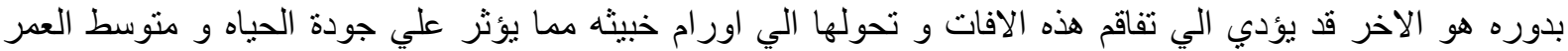

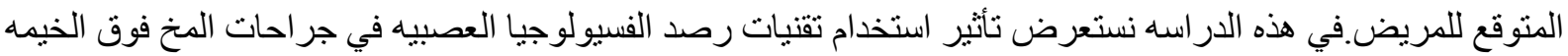
المخيه و تأثير ها علي مدي استنئصال الورم, و الحاله الوظيفيه للمريض بعد الجر احه.

هدف الدراسه: تقييم استخدام تقنيات رصد الفسيولوجيا العصبيه في جراحات استئصسال اورام المخ و البؤر الصرعيه علي مر اكز الحركه و الكلام, من حيث زياده نسبة الاستئصال و الحفاظ علي المر اكز الحيويه.

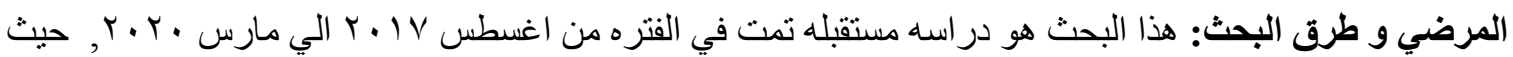

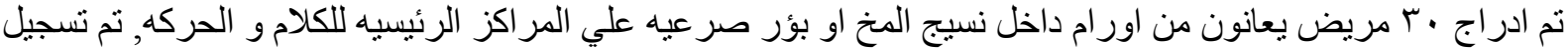

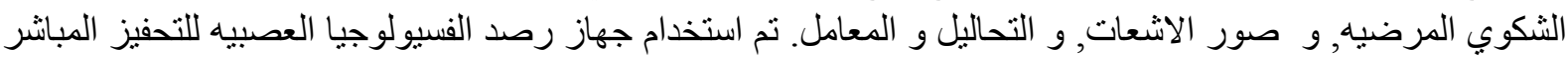

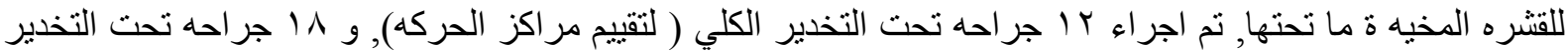

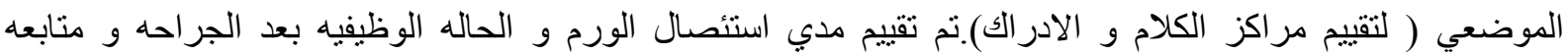

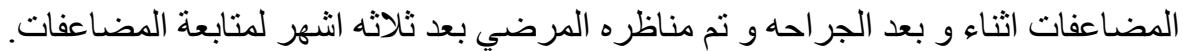

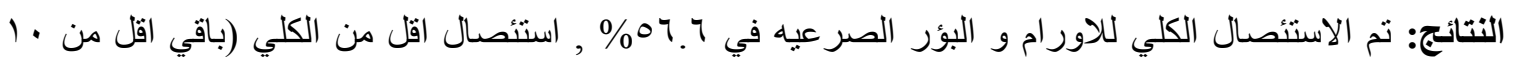

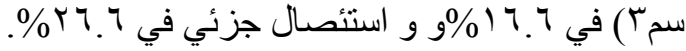

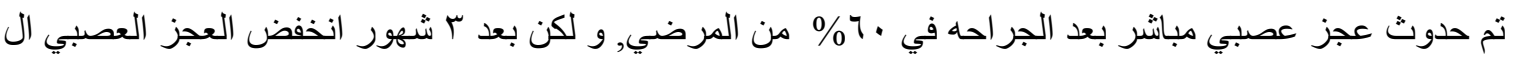
$\% 1 r . r$

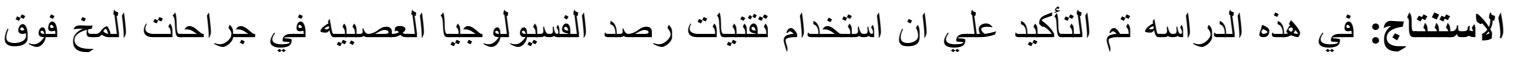

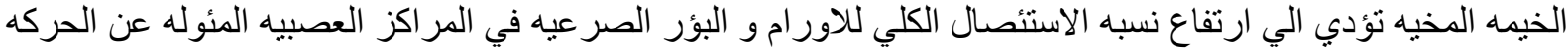

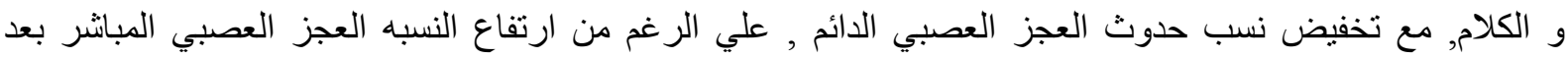
الجر احه. 